\title{
EXCREÇÃO FRACIONADA DO CÁLCIO E FÓSFORO EM NOVILHAS NELORE ANTES E DURANTE A GESTAÇÃO'1
}

\author{
ALEXANDRE SECORUN BORGES ${ }^{2}$, BRENO PELOSO DE BARROS ${ }^{3}$, ANA LIZ GARCIA ALVES ${ }^{2}$, \\ PAULO CÉSAR CIARLINE ${ }^{2}$, PEDRO LUIZ BICUDO ${ }^{4}$ e AGUEMI KOHAYAGAWA ${ }^{4}$
}

\begin{abstract}
RESUMO - A avaliação do cálcio e do fósforo nutricional é difícil quando se usam concentrações séricas desses elementos, pois estas permanecem dentro de valores normais apesar de variações do conteúdo desses minerais na dieta, pela alta eficiência do processo de homeostasia corporal. Alterações significativas serão observadas em processos carenciais crônicos, quando então as perdas econômicas já terão significado relevante. A técnica da excreção fracionada permite avaliação quantitativa sem a necessidade de determinação do volume urinário produzido. Com o objetivo de analisar a excreção fracionada de $\mathrm{Ca}$ e P, foram utilizadas 22 novilhas criadas extensivamente. As amostras de soro e urina foram colhidas a cada 45 dias, antes (duas coletas) e durante a gestação (seis coletas). A excreção fracionada do Ca e do $\mathrm{P}$ apresentou medianas variando entre $1,06 \%$ e $3,72 \%$ e $1,09 \%$ e $2,70 \%$, respectivamente. Houve tendência na diminuição dos valores da mediana da excreção fracionada do Ca, comparando-se os momentos em que os animais ainda não estavam gestantes com os valores obtidos durante a gestação. Este experimento demonstrou que existem amplas variações na excreção fracionada de $\mathrm{Ca}$ e $\mathrm{P}$ em diferentes animais em um mesmo momento, dificultando a utilização deste método na avaliação do estado nutricional desses elementos em bovinos criados extensivamente.
\end{abstract}

Termos para indexação: bovinos, metabolismo de minerais, urina, sangue, bioquímica, estado nutricional.

\section{CALCIUM AND PHOSPHORUS FRACTIONAL EXCRETION IN NELLORE BEFORE AND DURING PREGNANCY}

\begin{abstract}
The assessment of calcium and phosphorus nutrition is difficult when using serum concentrations of these elements, because they remain within normal ranges despite a wide variation in $\mathrm{Ca}$ and $\mathrm{P}$ contents in different diets due to the high efficiency of body homeostasis process. Significant alterations are observed in chronic deficiencies when economic losses have a relevant importance. The use of fractional excretion of $\mathrm{Ca}$ and $\mathrm{P}$ allow quantitative evaluation without need of timed urine volumetric determinations. Fractional excretion ratios for $\mathrm{Ca}$ and $\mathrm{P}$ were determined using eight consecutive 45-day urine and serum sample collections performed on 22 heifers before (two samplings) and during pregnancy (six samplings). The values presented by fractional excretion of $\mathrm{Ca}$ and $\mathrm{P}$ ranged from $1.06 \%$ to $3.72 \%$ and $1.09 \%$ to $2.70 \%$, respectively. There was a tendency to diminishing fractional excretion median values of $\mathrm{Ca}$ when comparing before pregnancy with values observed during pregnancy. This experiment showed that there is wide variation in $\mathrm{Ca}$ and $\mathrm{P}$ fractional excretion in different animals at the same moment and this results in difficulties to access nutritional status of these elements in cattle raised on pasture using this method.
\end{abstract}

Index terms: bovines, mineral metabolism, urine, blood, biochemistry, nutritional status.

${ }^{1}$ Aceito para publicação em 14 de janeiro de 2000. Trabalho financiado pela FUNDUNESP.

${ }^{2}$ Méd. Vet., M.Sc., Dep. de Clínica, Cirurgia e Reprodução Animal, UNESP, Rua Clóvis Pestana, 793, CEP 16050-680 Araçatuba, SP.

${ }^{3}$ Méd. Vet., M.Sc., Alameda Aquarius, s/n, Vale do Sol, CEP 18618-000 Botucatu, SP.

${ }^{4}$ Méd. Vet., Ph.D., Dep. de Clínica Veterinária, UNESP, Rua Rubião Junior, s/n, Caixa Postal 560, CEP 18618-000 Botucatu, SP.

\section{INTRODUÇÃO}

A criação de bovinos de corte é fundamental na economia do país. Assim, nutrição adequada e controle sanitário do rebanho são aspectos que não podem ser deixados de lado quando se objetiva criação eficiente. Com relação ao aspecto nutricional, deve-se prestar muita atenção ao fornecimento de minerais; segundo McDowell \& Conrad (1977), o 
fornecimento adequado de minerais é essencial para aumentar a produtividade do rebanho bovino.

O fornecimento de uma mistura mineral denominada adequada é uma variável difícil de ser avaliada, pois existem flutuações nas necessidades dos animais e também na composição mineral das plantas forrageiras. Dessa forma, alguns autores procuraram avaliar a eficiência de um suplemento mineral, levando em consideração os valores séricos de alguns eletrólitos, principalmente o Ca e o P. Tokarnia et al. (1970) observaram que bovinos recebendo nutrição mineral inadequada podem mostrar níveis séricos de $\mathrm{Ca}$ dentro de valores normais. Entretanto, o mesmo não foi observado em relação ao $\mathrm{P}$, em que os valores encontrados estavam abaixo do valor normal. Tais observações foram feitas em animais com carência crônica desses elementos. Balarin (1990), a partir de uma única amostragem de soro sangüíneo obtida de bovinos, a saber: de um grupo de animais com suplementação mineral adequada e, outro, com suplementação deficiente, não observou diferença significativa nos valores séricos de $\mathrm{Ca}$ e $\mathrm{P}$ entre os dois grupos. Segundo Boin (1985), a concentração de Ca no soro sangüíneo não apresenta grandes variações, pois a queda do nível de $\mathrm{Ca}$ no sangue aumenta a produção (secreção) do paratormônio, que estimula a síntese da vitamina $\mathrm{D}_{3}(1,25$ dihidroxicolecalciferol), diminuindo a excreção urinária de Ca.

Com o objetivo de utilizar um método mais sensível, Caple et al. (1982) concluíram, em seus estudos, que com amostras de urina é possível determinar de maneira prática se a dieta de eqüinos é baixa, adequada ou alta em $\mathrm{Ca}$ ou alta em P. Isto foi possível a partir da utilização dos valores séricos e urinários da creatinina, como indicador real da excreção de Ca e P. Ainda segundo esses autores, apenas o uso dos valores séricos de $\mathrm{Ca}$ e $\mathrm{P}$ não permite avaliação nutricional de tais elementos.

A aferição das concentrações de íons urinários ( $\mathrm{Ca}, \mathrm{P}, \mathrm{Na}, \mathrm{K}$ e $\mathrm{Cl}$ ) pode fornecer dados do balanço mineral por meio de quantificação da excreção desses elementos. Contudo, a simples dosagem da concentração dos eletrólitos urinários não pode ser corretamente interpretada sem que o volume urinário produzido seja considerado. A necessidade do conhecimento do volume urinário produzido não torna tal avaliação um método prático. Porém o volume urinário é diretamente proporcional à taxa de filtração glomerular, que pode ser estimada por meio do uso de uma substância que seja totalmente filtrada e não seja secretada ou absorvida pelos rins (King, 1994).

Neiger \& Hagemoser (1985) demonstraram que em bovinos, assim como em eqüinos, existe grande correlação entre a creatinina urinária e a densidade específica da urina, indicando, assim, que a creatinina é quase totalmente filtrada passivamente pelos glomérulos, e que são insignificantes as quantidades secretadas ou reabsorvidas pelos túbulos renais, fator importante para utilização dessa substância no cálculo da excreção fracionada em bovinos. Avaliando diferentes eletrólitos séricos, esses autores consideraram a creatinina adequada para cálculos da excreção fracionada desses eletrólitos. Segundo Fleming et al. (1991), a excreção fracionada de um eletrólito é o clearance desse eletrólito expresso em porcentual do clearance da creatinina. $\mathrm{O}$ cálculo da excreção fracionada de um eletrólito não requer o cálculo do volume urinário produzido em função do tempo, como ocorre no cálculo do clearance renal, pois o primeiro deles pode ser determinado pela coleta simultânea de uma amostra de sangue, e uma, de urina.

Balarin (1990), utilizando a creatinina nos cálculos para avaliar a excreção fracionada do $\mathrm{Ca}$ e do $\mathrm{P}$ em fêmeas bovinas adultas, observou desequilíbrio na excreção desses minerais no grupo de animais que não recebia mistura mineral. $\mathrm{O}$ autor apresentou valores da excreção fracionada do Ca de 2,06 $\pm 0,76 \%$ e 2,36 $\pm 1,84 \%$ em bovinos com deficiência e sem deficiência mineral, e de $1,75 \pm 1,08 \%$ e $0,97 \pm 0,78 \%$ de excreção fracionada do $\mathrm{P}$ em animais com e sem deficiência.

Neiger \& Hagemoser (1985) determinaram valores de $1,38 \pm 1,41 \%$ e de $15,6 \pm 14,3 \%$ da excreção fracionada de $\mathrm{P}$ e $\mathrm{Ca}$, respectivamente, em fêmeas bovinas da raça Holandesa, com idade variando entre 11 e 14 meses e alimentadas principalmente à base de concentrados.

Fleming et al. (1991), trabalhando com valores de excreção fracionada em bovinos submetidos a cateterização vesical, observou, em quatro análises diárias ( 0 a 6,6 a 12, 12 a 18 e 18 a 24 horas), valores 
de $1,02 \pm 0,79 \%, 0,84 \pm 0,32 \%, 0,63 \pm 0,29 \%$ e $0,65 \pm 0,34 \%$ referentes à excreção fracionada de $\mathrm{P}, \mathrm{e}$ de $1,29 \pm 2,78 \%, 0,82 \pm 1,22 \%, 1,09 \pm 2,26 \%$ e $0,95 \pm 1,88 \%$ referentes à excreção fracionada de $\mathrm{Ca}$, respectivamente. Não foram encontradas variações significativas entre os períodos do dia; porém, houve diferença $(\mathrm{p}<0,01)$ entre os animais estudados.

O objetivo deste trabalho foi determinar os valores da excreção fracionada do Ca e do $\mathrm{P}$ em novilhas da raça Nelore criadas extensivamente, antes e durante a gestação.

\section{MATERIAL E MÉTODOS}

Foram utilizadas 22 novilhas da raça Nelore com idade aproximada de 30 meses, de uma única fazenda, que permaneceram no mesmo piquete durante o decorrer do experimento. A alimentação era constituída exclusivamente de pasto de Brachiaria decumbens e suplemento mineral comercial fornecido ad libitum em cocho coberto. Os animais foram submetidos à monta natural, sendo a prenhez confirmada por palpação retal. Para efeito de controle sanitário os animais obedeceram a esquema de vermifugação e vacinação adotado na fazenda, que consiste em administração anual de vacina contra o botulismo, e duas administrações de vacina contra a aftosa. Os animais foram vermifugados duas vezes, durante este experimento, com produto contendo ivermectina.

Os animais foram avaliados durante a gestação, pois nessa fase ocorrem diferentes necessidades de $\mathrm{Ca}$ e $\mathrm{P}$, por causa da mobilização para o feto, e não foram encontrados valores na literatura no que se refere à excreção fracionada desses elementos em animais prenhes.

As amostras de sangue e urina foram coletadas antes (pré-gestação) e durante a gestação, sempre nos mesmos 22 animais, no período da manhã. Na etapa de pré-gestação foram realizadas duas coletas com intervalo de 45 dias, denominadas de amostras 1 e 2 . Após as coletas, os animais foram colocados em regime de monta natural. $\mathrm{Na}$ etapa de gestação foram feitas coletas a cada 45 dias, totalizando seis amostras, denominadas de amostras 3,4 , 5, 6, 7 e 8, correspondendo, portanto a: amostra 3 (entre 1 e 45 dias de gestação); amostra 4 (entre 46 e 90 dias de gestação); amostra 5 (entre 91 e 135 dias de gestação); amostra 6 (entre 136 e 180 dias de gestação); amostra 7 (entre 181 e 225 dias de gestação), e amostra 8 (entre 226 e 270 dias de gestação).

Para análises bioquímicas foram coletados, imediatamente após a coleta da urina, aproximadamente $30 \mathrm{~mL}$ de sangue de cada animal, por punção da jugular. O sangue foi colhido em tubos previamente lavados com detergente $\mathrm{e}$ solução ácida e enxaguados com água deionizada, com capacidade de $15 \mathrm{~mL}$ e submetidos à centrifugação, em no máximo, 30 minutos após a coleta. O soro obtido foi conservado congelado $\left(-20^{\circ} \mathrm{C}\right)$ até a realização dos exames.

As amostras de urina foram colhidas mediante massagem da região perineal e armazenadas em frascos de vidro lavados com detergente e solução ácida e enxaguados com água deionizada. As amostras de urina foram imediatamente refrigeradas e analisadas no máximo 12 horas após a coleta. O volume obtido ficou ao redor de $100 \mathrm{~mL}$ por amostra.

Todas as dosagens bioquímicas no soro foram realizadas utilizando-se reagentes comerciais da marca Labtest; cada amostra foi submetida a uma repetição; foi utilizada a média dos dois valores. Quando a diferença entre os dois resultados obtidos na mesma amostra foi superior à descrita nas recomendações presentes, nos reagentes comerciais, o procedimento era novamente repetido. Para controle de cada dosagem, foi utilizado padrão fornecido em cada reagente. Os seguintes métodos foram utilizados para a dosagem sérica: $\mathrm{Ca}$ (método da púrpura de ftaleína); $\mathrm{P}$ inorgânico (reação com o molibdênio); creatinina (método do picrato); proteína total (reação com o biureto); albumina (reação com o verde de bromocresol).

A urina foi submetida à urinálise tipo 1 (cor, odor, $\mathrm{pH}$, aspecto, densidade, glicose, proteínas e exame do sedimento), para descartar animais com anormalidades. Foram também realizadas análises bioquímicas da urina com o objetivo de determinar a creatinina, $\mathrm{Ca}$ e $\mathrm{P}$. As análises foram realizadas com o auxílio de kits da mesma marca citada anteriormente para dosagens séricas. As dosagens de $\mathrm{Ca}$ e $\mathrm{P}$ foram realizadas após prévia acidificação das amostras, segundo técnica descrita por Fleming et al. (1991).

Para o cálculo da excreção fracionada utilizou-se a seguinte equação (Fleming et al., 1991):

\footnotetext{
Excreção Fracionada $(\%)=(\mathrm{Eu} / \mathrm{Es}) \times(\mathrm{Crs} / \mathrm{Cru}) \times 100$

em que

$\mathrm{Eu}=$ concentração urinária do eletrólito;

Es = concentração sérica do eletrólito;

Crs = concentração sérica da creatinina;

Cru = concentração urinária da creatinina.
}

$\mathrm{Na}$ análise estatística das variáveis quantitativas, cuja tendência central pode ser mais bem caracterizada pela mediana, utilizou-se a prova não-paramétrica de Friedman (Zar, 1984), com o cálculo da estatística qui-quadrado $\left(\chi^{2}\right)$ e de seu respectivo valor de $\mathrm{p}$. 


\section{RESULTADOS E DISCUSSÃO}

A mediana dos valores da excreção fracionada do $\mathrm{Ca}$ foi diminuindo gradativamente, excetuando-se as coletas de número 5 e 8 , mas apenas as coletas de número 1 e 6, 2 e 6, 2 e 7 diferiram $(\mathrm{P}<0,05)$. Houve tendência de diminuição dos valores da excreção fracionada do $\mathrm{Ca}$ no decorrer do experimento, havendo portanto a possibilidade de os valores da excreção fracionada estarem associados à gestação (Tabela 1).

$\mathrm{O}$ menor valor da excreção fracionada do $\mathrm{Ca}$ foi de $0,23 \%$, encontrado no animal de número $11 \mathrm{du}$ rante a quinta coleta, e o maior valor foi de $15,90 \%$, encontrado no animal de número 21 durante a tercei- ra coleta (Tabela 1 ). Os valores apresentaram variação entre as diferentes coletas referentes ao mesmo animal, e assim, não foi possível estabelecer um padrão. O alto coeficiente de variação dessas coletas deve-se, em grande parte, à alta oscilação dos valores da creatinina urinária e do Ca urinário. Os valores médios da excreção fracionada do Ca apresentaramse mais elevados do que os relatados por Neiger \& Hagemoser (1985). Esses valores também foram mais elevados do que os encontrados por Fleming et al. (1991), porém com coeficiente de variação semelhante. Comparando-se tais resultados com os obtidos por Balarin (1990), pode-se constatar similaridade muito grande com o grupo de fêmeas sem deficiência mineral.

TABELA 1. Valores de excreção fracionada do cálcio (\%) em bovino, em oito coletas com intervalo de 45 dias ${ }^{1}$.

\begin{tabular}{|c|c|c|c|c|c|c|c|c|}
\hline \multirow[t]{2}{*}{$\mathrm{N}$} & \multicolumn{8}{|c|}{ Coleta $^{2}$} \\
\hline & 1 & 2 & 3 & 4 & 5 & 6 & 7 & 8 \\
\hline 1 & 1,38 & 4,42 & 4,44 & 1,26 & 1,98 & 1,40 & 0,41 & 2,69 \\
\hline 2 & 8,71 & 4,53 & 5,39 & 0,78 & 3,23 & 1,56 & 3,48 & 2,71 \\
\hline 3 & 1,30 & 1,22 & 1,53 & 4,02 & 0,57 & 1,71 & 1,65 & 4,50 \\
\hline 4 & 10,67 & 2,25 & 1,20 & 0,65 & 1,65 & 1,68 & 0,46 & 9,91 \\
\hline 5 & 6,97 & 14,92 & 5,10 & 1,02 & 0,96 & 1,28 & 1,13 & 3,30 \\
\hline 6 & 1,61 & 2,84 & 3,34 & 1,30 & 0,38 & 0,86 & 1,08 & 5,51 \\
\hline 7 & 5,04 & 6,08 & 2,47 & 4,06 & 9,14 & 1,07 & 9,29 & 1,63 \\
\hline 8 & 1,49 & 1,97 & 1,04 & 0,70 & 3,34 & 1,41 & 5,27 & 4,02 \\
\hline 9 & 1,43 & 12,47 & 2,72 & 1,58 & 2,13 & 0,56 & 1,18 & 4,52 \\
\hline 10 & 1,11 & 3,48 & 3,54 & 1,10 & 2,37 & 1,95 & 1,91 & 0,92 \\
\hline 11 & 0,65 & 0,78 & 1,15 & 0,70 & 0,23 & 0,74 & 0,28 & 2,61 \\
\hline 12 & 2,70 & 3,30 & 3,40 & 1,32 & 0,34 & 1,53 & 0,74 & 3,93 \\
\hline 13 & 2,86 & 1,14 & 7,73 & 4,78 & 0,90 & 3,03 & 6,98 & 6,16 \\
\hline 14 & 3,07 & 3,49 & 2,69 & 1,15 & 0,66 & 1,15 & 1,93 & 1,65 \\
\hline 15 & 2,75 & 3,39 & 2,07 & 1,83 & 1,59 & 1,16 & 1,19 & 10,24 \\
\hline 16 & 7,92 & 5,71 & 2,00 & 1,51 & 0,62 & 0,90 & 2,45 & 4,22 \\
\hline 17 & 8,20 & 6,27 & 9,40 & 5,71 & 7,49 & 0,49 & 6,05 & 4,53 \\
\hline 18 & 2,04 & 0,77 & 0,34 & 0,70 & 1,06 & 3,40 & 8,47 & 4,27 \\
\hline 19 & 3,05 & 1,39 & 1,10 & 2,40 & 0,91 & 0,89 & 1,02 & 1,69 \\
\hline 20 & 3,77 & 1,49 & 3,31 & 0,71 & 0,58 & 2,21 & 3,00 & 3,87 \\
\hline 21 & 0,97 & 2,14 & 15,90 & 1,39 & 1,11 & 1,00 & 3,57 & 1,65 \\
\hline 22 & 0,54 & 2,86 & 4,92 & 1,00 & 1,28 & 0,47 & 1,55 & 1,61 \\
\hline Média & 4,02 & 4,54 & 2,73 & 2,48 & 3,06 & 1,38 & 1,99 & 3,33 \\
\hline Mediana & 3,72 & 3,06 & 2,59 & 1,79 & 1,90 & 1,06 & 1,17 & 2,70 \\
\hline S & 2,92 & 3,62 & 1,79 & 2,27 & 3,55 & 1,44 & 2,02 & 2,68 \\
\hline $\mathrm{CV}$ & 72,56 & 79,72 & 65,73 & 91,25 & 116,06 & 105,01 & 101,56 & 80,31 \\
\hline
\end{tabular}


As medianas da excreção fracionada do P durante o experimento e os valores individuais em cada animal durante as diferentes coletas encontram-se na Tabela 2. A excreção fracionada do P apresentou mediana com valores variando de $1,09 \%$ a $2,70 \%$, e houve diferença $(\mathrm{P}<0,05)$ entre as medianas das coletas 1 e 8, 4 e 7, 4 e 8, 5 e 8, 6 e 8 . Esses valores foram mais elevados do que os obtidos por Balarin (1990) em animais normais e em animais deficientes em $\mathrm{Ca} e$ P. O presente trabalho foi realizado com animais em bom estado nutricional e recebendo sal mineral de boa qualidade. $\mathrm{O}$ aumento nos valores da mediana da excreção fracionada do P na última coleta devese, em parte, à diminuição das medianas dos valores de $\mathrm{P}$ sérico em relação às outras coletas. Os valores da excreção fracionada do P são inferiores aos de
Neiger \& Hagemoser (1985), que apresentaram média de 15,6\%, obtidos de animais alimentados com concentrados, o que, segundo Scott (1972), aumenta a excreção do P na urina.

Outra justificativa para as grandes variações está no fato de que a principal via de excreção do P seria o trato gastrintestinal (Kleiber \& Smith, 1951). Isto não impediria a utilização desse procedimento em bovinos. Neiger \& Hagemoser (1985) sugerem que animais suplementados com alimentos ricos em $\mathrm{P}$, porém de baixa disponibilidade, podem ser monitorados pela excreção fracionada do P. Fleming et al. (1991) apresentam resultados mais baixos para a excreção fracionada do $\mathrm{P}$, semelhantes aos valores apresentados por Balarin (1990), cujo valor mais alto em quatro coletas foi de $1,02 \pm 0,79 \%$, e o mais baixo,

TABELA 2. Valores de excreção fracionada do fósforo (\%) em bovino, em oito coletas com intervalo de 45 dias ${ }^{1}$.

\begin{tabular}{|c|c|c|c|c|c|c|c|c|}
\hline \multirow[t]{2}{*}{$\mathrm{N}$} & \multicolumn{8}{|c|}{ Coleta $^{2}$} \\
\hline & 1 & 2 & 3 & 4 & 5 & 6 & 7 & 8 \\
\hline 1 & 2,15 & 1,58 & 3,07 & 0,87 & 1,60 & 0,93 & 3,80 & 3,83 \\
\hline 2 & 2,85 & 1,25 & 1,77 & 1,30 & 1,09 & 2,50 & 1,67 & 4,98 \\
\hline 3 & 0,63 & 2,67 & 1,00 & 0,35 & 1,01 & 1,58 & 3,01 & 2,29 \\
\hline 4 & 14,60 & 2,66 & 2,10 & 1,19 & 1,80 & 1,72 & 1,86 & 5,13 \\
\hline 5 & 4,97 & 8,62 & 1,47 & 1,28 & 1,07 & 1,78 & 1,77 & 2,98 \\
\hline 6 & 2,09 & 1,64 & 1,13 & 0,98 & 0,99 & 1,88 & 1,37 & 3,35 \\
\hline 7 & 0,50 & 1,64 & 1,87 & 1,66 & 12,06 & 1,35 & 2,59 & 4,06 \\
\hline 8 & 2,47 & 1,65 & 2,14 & 0,88 & 1,71 & 1,94 & 2,32 & 3,29 \\
\hline 9 & 1,18 & 15,45 & 2,31 & 1,72 & 2,98 & 2,13 & 1,43 & 2,04 \\
\hline 10 & 0,67 & 2,22 & 2,10 & 0,95 & 4,74 & 1,83 & 2,89 & 1,60 \\
\hline 11 & 1,26 & 1,01 & 1,25 & 0,88 & 1,37 & 2,25 & 2,21 & 2,46 \\
\hline 12 & 2,33 & 1,68 & 4,36 & 1,27 & 0,76 & 1,30 & 1,34 & 2,06 \\
\hline 13 & 0,61 & 2,01 & 0,78 & 1,11 & 1,66 & 1,10 & 5,19 & 1,87 \\
\hline 14 & 1,22 & 1,83 & 4,48 & 1,34 & 0,92 & 1,53 & 0,98 & 3,52 \\
\hline 15 & 0,85 & 1,81 & 1,24 & 1,66 & 1,05 & 1,18 & 1,28 & 2,89 \\
\hline 16 & 2,33 & 1,88 & 1,35 & 1,66 & 0,91 & 0,53 & 1,81 & 4,00 \\
\hline 17 & 1,79 & 2,95 & 1,83 & 1,67 & 11,88 & 1,96 & 1,94 & 2,52 \\
\hline 18 & 0,62 & 0,94 & 0,97 & 1,61 & 0,91 & 1,90 & 1,90 & 1,83 \\
\hline 19 & 1,01 & 1,20 & 2,33 & 1,55 & 1,14 & 2,39 & 2,53 & 1,77 \\
\hline 20 & 1,35 & 1,93 & 8,30 & 1,58 & 0,92 & 1,38 & 1,62 & 5,25 \\
\hline 21 & 0,82 & 1,28 & 18,68 & 2,13 & 0,63 & 1,85 & 1,65 & 1,73 \\
\hline 22 & 1,00 & 1,43 & 6,88 & 1,31 & 1,09 & 0,95 & 2,37 & 1,94 \\
\hline Média & 2,15 & 2,70 & 3,25 & 1,32 & 2,38 & 1,63 & 2,16 & 2,97 \\
\hline Mediana & 1,24 & 1,74 & 1,98 & 1,30 & 1,09 & 1,75 & 1,88 & 2,70 \\
\hline S & 2,97 & 3,24 & 3,95 & 0,40 & 3,23 & 0,50 & 0,95 & 1,16 \\
\hline $\mathrm{CV}$ & 138,05 & 120,23 & 121,56 & 30,3 & 135,94 & 30,84 & 43,78 & 39,19 \\
\hline
\end{tabular}

${ }^{1} \mathrm{~N}$ : número do animal; S: desvio-padrão; CV: coeficiente de variação.

2 1: animais não-gestantes; 2: animais não-gestantes, 45 dias após a primeira coleta; 3: entre 1 e 45 dias de gestação; 4 : entre 46 e 90 dias de gestação; 5: entre 91 e 135 dias de gestação; 6: entre 136 e 180 dias de gestação; 7: entre 181 e 225 dias de gestação; 8: entre 226 e 270 dias de gestação. 
de $0,65 \pm 0,34 \%$. As coletas $1,2,3$ e 5 apresentaram correlação positiva e significativa entre os valores da excreção fracionada do Ca e P.

As grandes variações na excreção fracionada do $\mathrm{Ca}$ e $\mathrm{P}$, em diferentes animais, em determinado momento, resultam em grande dificuldade na interpretação do balanço de $\mathrm{Ca}$ e $\mathrm{P}$ de forma individual em bovinos criados extensivamente.

\section{CONCLUSÕES}

1. Ocorre diminuição dos valores das medianas da excreção fracionada do cálcio comparando-se os momentos antes da gestação com os momentos durante a gestação.

2. As amplas variações na excreção fracionada de cálcio e de fósforo sugerem que se deve fazer uma interpretação cuidadosa dos valores obtidos e a serem utilizados para a avaliação do estado nutricional em bovinos criados extensivamente.

\section{AGRADECIMENTOS}

À administração da Fazenda Natal, Caiuá, SP, por ter possibilitado a realização deste experimento mediante a utilização de suas instalações e animais; aos seus funcionários, pela grande colaboração durante o período de um ano; e ao Prof. Dr. Paulo Cury, pela realização da análise estatística.

\section{REFERÊNCIAS}

BALARIN, N.R.S. Avaliação do estado nutricional de cálcio e fósforo em bovinos por meio da análise bioquímica da urina. Botucatu : UNESP-Faculdade de Medicina Veterinária e Zootecnia, 1990. 34p. Dissertação de Mestrado.

BOIN, C. Exigências de minerais pelas categorias do rebanho bovino e funções desses nutrientes. In:
SIMPÓSIO SOBRE NUTRIÇÃO DE BOVINOS, 3., 1985, Piracicaba. Anais. Piracicaba : FEALQ, 1985. p.15-19.

CAPLE, I.W.; DOAKE, P.A.; ELLIS, P.G. Assessment of the calcium and phosphorus nutrition in horses by analysis of urine. Australian Veterinary Journal, Artarmon, v.58, n.4, p.125-131, 1982.

FLEMING, S.A.; HUNT, E.L.; RIVIERE, J.E.; ANDERSON, K.L. Renal "clearance" and fractional excretion of electrolytes over four 6-hour periods in cattle. American Journal of Veterinary Research, Schaumburg, v.52, n.1, p.5-8, 1991.

KING, C. Practical use of urinary fractional excretion. Journal of Equine Veterinary Science, Wildomar, v.14, n.9, p.464-468, 1994.

KLEIBER, M.; SMITH, A.H. Radiophosphorus $\left(\mathrm{P}^{32}\right)$ as tracer for measuring endogenous phosphorus in cows feces. Journal of Nutrition, Bethesda, v.45, p.253263, 1951

McDOWELL, L.R.; CONRAD, J.H. Trace mineral nutrition in Latin America. World Animal Review, Rome, n.24, p.24-33, 1977.

NEIGER, R.D.; HAGEMOSER, W. Renal percent "clearance" ratios in cattle. Veterinary Clinical Pathology, Mission Viejo, v.14, n.1, p.31-35, 1985.

SCOTT, D. Excretion of phosphorus and acid in the urine of sheep and calves fed either roughage or concentrate diets. Quarterly Journal of Experimental Physiology, Cambridge, Grã-Bretanha, v.57, n.4, p.379-392, 1972.

TOKARNIA, C.H.; CANELLA, C.F.C.; GUIMARÃES, J.A.; DÖBEREINER, J.; LANGENEGGER, J. Deficiência de fósforo em bovinos no Piauí. Pesquisa Agropecuária Brasileira, Rio de Janeiro, v.5, p.483494, 1970.

ZAR, J.H. Biostatistical analysis. 2.ed. Englewood Cliffs : Prentice Hall, 1984. 718p. 